\title{
Caracterização estrutural e status de conservação do estrato herbáceo de dunas da Praia de São Marcos, Maranhão, Brasil
}

\author{
Ana Cássia Medeiros ARAUJO ${ }^{1}$, Ariade Nazaré Fontes da SILVA², Eduardo Bezerra de ALMEIDA Jr. ${ }^{3 *}$ \\ 1 Universidade Federal do Maranhão, Programa de Pós-Graduação em Biodiversidade e Conservação, Departamento de Biologia, Avenida dos Portugueses, 1966, Bacanga, \\ CEP 65080-805, São Luís, MA, Brasil. \\ 2 Universidade Federal do Maranhão, Laboratório de Estudos Botânicos, Avenida dos Portugueses, 1966, Bacanga, CEP 65080-805, São Luís, MA, Brasil. \\ 3 Universidade Federal do Maranhão, Laboratório de Estudos Botânicos, Avenida dos Portugueses, 1966, Bacanga, CEP 65080-805, São Luís, MA, Brasil. \\ Autor correspondente: ebaj25@yahoo.com.br
}

\section{RESUMO}

As dunas costeiras têm sido apontadas como um dos ambientes mais deteriorados do litoral brasileiro ameaçando assim a vegetação de restinga. O objetivo desse estudo foi descrever a fitossociologia do estrato herbáceo da vegetação de dunas e avaliar o status de conservação da área no litoral maranhense. O estudo foi realizado na Praia de São Marcos, localizada na porção norte da Ilha do Maranhão. Para a amostragem fitossociológica foi utilizado o método de parcelas (50 parcelas de 1 $\mathrm{m}^{2}$ ), sendo analisados os parâmetros fitossociológicos tradicionais em vegetação. Também foram determinadas as formas de vida, distribuição fitogeográfica e status de conservação da comunidade vegetal. Nos $50 \mathrm{~m}^{2}$ foram amostradas 35 espécies das quais Paspalum maritimum, Paspalum ligulare, Cassytha filiformis, Chamaecrista hispidula e Euploca polyphylla obtiveram os maiores VI's. O índice de diversidade de Shannon, quando calculado com valores de cobertura, resultou em H' =1,69 nats m ${ }^{-2}$ e quando calculado com valores de frequência, foi de $\mathrm{H}^{\prime}=3,04$ nats $\mathrm{m}^{-2}$. A área exibiu predominância de caméfitos e terófitos, indicando que essas formas de vida são uma estratégia adaptativa das espécies que ocorrem nesse tipo de vegetação para resistir as condiçóes climáticas adversas. $\mathrm{O}$ ecossistema amostrado apresentou espécies de ampla distribuição nos biomas brasileiros, sendo ausentes endemismos. Foram identificados 14 fatores de impactos, dos quais cinco atingem com mais gravidade a vegetação de restinga. Portanto, são necessários mais estudos com foco na conservação dos ecossistemas de dunas, a fim de evitar danos que comprometam a diversidade vegetal dessas áreas.

PALAVRAS-CHAVE: Fitossociologia, Restinga, Antropização, Litoral maranhense

\section{Structural characterization and conservation status of the herbaceous stratum at Praia de São Marcos dunes, Maranhão State, Brazil}

\section{ABSTRACT}

The coastal dunes have been identified as one of the most degraded environments. On the Brazilian coast, they threat the "restinga" vegetation. The objective of this study was to describe the phytosociological structure of the herb layer of dune vegetation and assess the conservation status of the area on the Maranhão coast. The study was conducted at São Marcos Beach located in the northern portion of the Maranhão Island. For the phytosociological sampling, we used the plot's method (50 plots of $1 \mathrm{~m}^{2}$ ), and analyzed the traditional phytosociological parameters of vegetation. Also, there were determined the life forms, phytogeographic distribution and conservation status of vegetation community on site. In the sample of $50 \mathrm{~m}^{2}$ we found 35 species from which Paspalum maritimum, Paspalum ligulare, Cassytha filiformis, Chamaecrista hispidula and Euploca polyphylla showed the highest importance value. When calculated for the cover values, the Shannon diversity index $\left(\mathrm{H}^{\prime}\right)$ was 1.69 nats $\mathrm{m}^{-2}$ and it was 3.04 nats $\mathrm{m}^{-2}$ when calculated for the frequency values. The area showed predominance of chamaephytes and therophytes indicating an adaptive strategy of vegetation to withstand adverse weather conditions. The sampled ecosystem presented widely distributed species in the Brazilian biomes, being absent endemism. There were identified 14 impacts factors of which five hit more severely the restinga vegetation of the São Marcos Beach. Therefore, more studies are needed with a focus on conservation of dune ecosystems to prevent damage compromising the plant diversity of these areas.

KEYwORDS: Phytosociology, Restinga vegetation, Anthropization, Maranhão's Coast 


\section{INTRODUÇÃO}

As dunas se destacam na costa brasileira como um dos ambientes da zona costeira mais degradados pela antropizaçáo (Carboni et al. 2009) sofrendo continuamente uma série de impactos antrópicos que alteram sua dinâmica e comprometem sua biodiversidade (Santos e Medeiros 2003; Oliveira e Sousa 2011).

Almeida e Suguio (2012) sinalizam para os impactos aos quais as dunas estão submetidas citando, entre eles, a remoção da cobertura vegetal natural, o que culmina na reativação de processos de migraçáo da duna, posto que a vegetaçáo colonizadora deste ambiente exerce papel fundamental na sua fixação e manutenção (Leite e Andrade 2004).

A vegetação herbácea é um importante componente na estabilizaçáo de dunas, principalmente das porçôes mais próximas ao mar (Cabral-Freire e Monteiro 1993; Falkenberg 1999; Cordazzo et al. 2006). Porém, em situaçôes de estresse ambiental, a comunidade herbácea é a primeira a manifestar modificações (Citadini-Zanette e Baptista 1989). Um problema ligado aos estudos de vegetação de dunas é que muitos desses ecossistemas são dominados por plantas herbáceas, possuindo uma vegetação campestre. Entretanto, há uma escassez de dados referentes à estrutura fitossociológica do estrato herbáceo em diversos tipos vegetacionais (Pereira $e t$ al. 2004; Inacio e Jarenkow 2008; Munhoz e Araújo 2011). Estes estudos fitossociológicos podem servir de referência para projetos de recuperaçáo ambiental, registrando a composição e estrutura da vegetação antes que as áreas sejam alteradas, uma vez que vários pontos da zona costeira estão sendo suprimidos em razão da urbanização do litoral (Silva 1999).

Ao longo do litoral brasileiro são escassos estudos que tratam da caracterização fitossociológica do estrato herbáceo na fisionomia de dunas, citando-se apenas Danilevicz et al. (1990) em Santa Catarina, Cordeiro (2005) no Rio de Janeiro e Palma e Jarenkow (2008) no Rio Grande do Sul. Para o litoral nordestino aponta-se somente o estudo de Menezes et al. (2012) no litoral da Bahia.

Em São Luís, particularmente, a especulação imobiliária é um agravante da depredação destas áreas, com a retirada de paleodunas e dunas, além da construção e prolongamento de uma avenida ao longo da orla (Cabral-Freire e Monteiro 1994; Silva 2012).

Em contextos de elevada perturbação antrópica, estudos fitossociológicos se sobressaem por fornecerem subsídios para a compreensão da estrutura e dinâmica de formaçóes vegetais (Chaves et al. 2013). Desta forma, ressalta-se a importância de abordagens fitossociológicas e de estudos que tracem panoramas do status de conservaçáo em dunas na Costa nordestina, em especial, no litoral maranhense, visto a escassez de dados estruturais da comunidade vegetal de dunas, resultando em grandes lacunas acerca da distribuição, diversidade e conservação de espécies herbáceas diante das ameaças iminentes a esse ecossistema. Diante deste contexto, este estudo tem como objetivo descrever a estrutura da vegetação herbácea e apresentar o status de conservação das Dunas da Praia de São Marcos.

\section{MATERIAL E MÉTODOS}

\section{Área de estudo}

O estudo foi realizado nas dunas da Praia de Sáo Marcos, localizadas na Ilha do Maranhão, município de Sáo Luís $\left(02^{\circ} 29^{\prime} 07^{\prime \prime}\right.$ ' $\left.44^{\circ} 15^{\prime} 59^{\prime \prime} \mathrm{W}\right)$. A área apresenta aproximadamente $4 \mathrm{~km}$ de extensão (Figura 1).

O clima para a região é do tipo Aw, possuindo duas estaçóes distintas (Köppen 1948) uma chuvosa que compreende o período que se estende de janeiro a junho e, a outra seca, no período de julho a dezembro. Segundo dados do Instituto Nacional de Meteorologia (INMET 2015), que compreendem o período de 1961 a 2009 , a temperatura média é de $26^{\circ}$ a $27^{\circ}$ $\mathrm{C}$ e a precipitaçáo anual varia de 1.250 a $2.000 \mathrm{~mm}$.

A regiáo norte da Ilha de Sáo Luís compreende praias arenosas com dunas e pequenos cursos de água doce que apresentam manchas de mangue (Cabral-Freire e Monteiro 1993). A Praia de São Marcos caracteriza-se por extensas áreas de dunas secundárias, as quais se situam nas zonas mais interiores, com relevo íngreme e alturas elevadas, e por dunas primárias, as quais ocorrem próximas à faixa de praia, perturbadas pela avenida de acesso principal (Cabral-Freire e Monteiro 1994).

\section{Amostragem fitossociológica}

Para análise estrutural da vegetação foi utilizado o método de parcelas múltiplas de $1 \mathrm{~m}^{2}$ (Mueller-Dombois e Ellenberg 1974; Munhoz e Araújo 2011). Ao longo da extensão das dunas da Praia de São Marcos foram alocadas transeçóes distantes $10 \mathrm{~m}$ entre si e perpendiculares à linha da praia. Em cada transeçấo foram demarcadas, a cada $10 \mathrm{~m}$, parcelas de $1 \mathrm{~m}^{2}$, plotadas à esquerda ou à direita, conforme sorteio prévio. O número de parcelas diferiu entre as transeçóes devido à faixa de vegetação herbácea divergir quanto à extensão e a área de estudo apresentar irregularidades. Por efetivamente não representarem a fitofisionomia da vegetação de dunas, foram desconsideradas da amostragem as transeçóes alocadas em trechos da vegetação cuja maior parte se encontrava fortemente alterada por ação de fogo ou formada por espécies cultivadas pelo homem para contenção da duna.

Como pertencentes ao estrato herbáceo foram consideradas ervas, subarbustos e trepadeiras (Veloso et al. 1991). A suficiência amostral foi determinada por meio da curva de acumulação de espécies por parcela amostrada. 


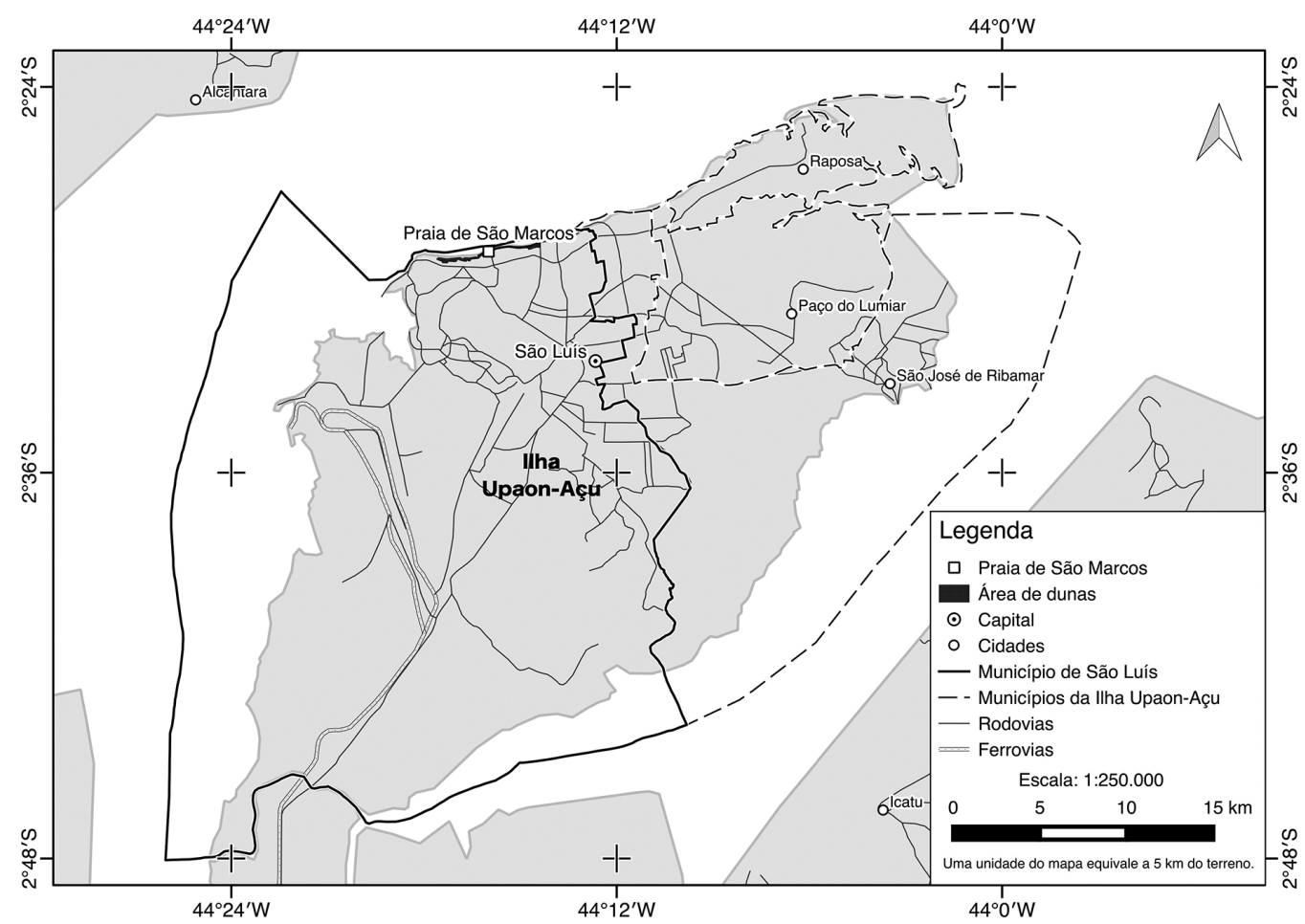

Figura 1. Localização geográfica das dunas da Praia de São Marcos (área de amostragem), São Luís, Maranhão.

A área de cobertura vegetal das espécies encontradas nas parcelas foi estimada visualmente (Brower e Zar 1977) com um auxílio de um quadrado de $1 \mathrm{~m}^{2}$, dividido em 100 quadrados menores de $10 \mathrm{~cm} \times 10 \mathrm{~cm}$ correspondente a $1 \%$ da área, disposto acima do solo.

Os parâmetros fitossociológicos de cobertura e frequência (absoluta e relativa) e valor de importância (VI) foram calculados para cada espécie (Mueller-Dombois e Ellenberg 1974) utilizando o programa Microsoft EXCEL ${ }^{\oplus} 2010$. O valor de importância (VI) foi obtido a partir do somatório dos valores de frequência e cobertura relativas, excluindo-se, portanto, o parâmetro densidade. Tal prática vem sendo utilizada para plantas herbáceas por alguns autores devido à dificuldade de individualizaçáo de espécies rizomatosas e estoloníferas (Cordeiro 2005; Carvalho e Sá 2011).

A estrutura da comunidade foi determinada segundo Carvalho e Sá (2011) adaptado de Pereira et al. (2004), a partir da classificaçấo das espécies por meio do valor de importância, em que espécies cujo VI $>10$ foram consideradas dominantes, espécies com $10>\mathrm{VI}>1$ foram consideradas intermediárias e espécies cujo VI $<1$ foram consideradas raras.

A diversidade foi calculada adotando o índice de diversidade de Shannon $\left(\mathrm{H}^{\prime}\right)$ e a uniformidade através da equabilidade de Pielou (J'). Para ambos os índices foram utilizados os dados de cobertura e frequência das espécies como medida de abundância (Magurran 1988).

\section{Identificação do material botânico}

Um indivíduo de cada espécie foi coletado (como material testemunho) para herborizaçáo, seguindo metodologia proposta por Mori et al. (1989). As exsicatas produzidas encontram-se depositadas no acervo do Herbário do Maranhão (MAR) do Departamento de Biologia da Universidade Federal do Maranhão.

A identificação taxonômica foi realizada a partir de chaves de identificação e comparação com material de Herbários. A listagem das famílias seguiu a proposta de classificação do APG III (2009). As formas de vida das espécies foram determinadas a partir de observaçóes posteriores em campo e consulta à literatura especializada, seguindo a classificação de Raunkiaer (1934).

A distribuição das espécies nos principais biomas brasileiros (Amazônia, Caatinga, Cerrado, Floresta Atlântica, Pampas e Pantanal) foi verificada segundo dados da Lista de Espécies da Flora do Brasil (2015) e consultas à literatura especializada.

\section{Avaliação do status de conservação da área}

O status de conservaçáo da área de estudo foi avaliado a partir da identificação de quais impactos antrópicos ocorrem na área, com registro dos impactos de maior e menor ocorrência. Os impactos foram levantados de acordo com a lista de categorias de impactos propostas por Rocha et al. (2003) para áreas de Restinga, sendo contemplada nesta análise toda a extensão das dunas da Praia de São Marcos. 


\section{RESULTADOS}

Foram encontradas 35 espécies, distribuídas em 28 gêneros pertencentes a 16 famílias (Tabela 1). As famílias que apresentaram maior número de espécies foram Fabaceae com 10 espécies, seguida por Asteraceae, Poaceae e Rubiaceae, com três espécies cada e Cyperaceae, Malvaceae e Turneraceae, com duas espécies cada. Mais da metade $(56 ; 2 \%)$ das famílias levantadas no presente estudo foi representada, apenas, por uma espécie (Figura 2).
A curva de acumulação de espécies estabilizou-se a partir da parcela 41, onde o número de 35 espécies foi atingido e mantido (Figura 3). Desta forma, o tamanho da amostra (50 parcelas de $1 \mathrm{~m}^{2}$ ) foi considerado suficiente para determinar a comunidade herbácea nestas dunas.

A análise fitossociológica conferiu maior valor de importância para as espécies Paspalum maritimum (54\%), Paspalum ligulare (20;8\%), Cassytha filiformis (20;4\%),

Tabela 1. Espécies amostradas e forma de vida do estrato herbáceo das dunas da Praia de São Marcos, São Luís, Maranhão, juntamente com a distribuição nos principais domínios fitogeográficos brasileiros e parâmetros fitossociológicos. (Formas de Vida: $\mathrm{Hc}=$ hemicriptófito; $\mathrm{Te}=\mathrm{terófito;} \mathrm{Cf}=\mathrm{caméfito}$; $\mathrm{Tr}=$ trepadeira; $\mathrm{Hp}=$ holoparasita. Biomas brasileiros: $\mathrm{AM}=$ Amazônia; $\mathrm{CAA}=$ Caatinga; $\mathrm{CR}=$ Cerrado; $\mathrm{FA}=$ Floresta Atlântica; $\mathrm{PA}=\mathrm{Pampa} ; \mathrm{PN}=\mathrm{Pantanal}$. Parâmetros fitossociológicos: $\mathrm{NP}=$ número de parcelas com ocorrência da espécie; $\mathrm{CA}=$ cobertura absoluta da espécie (\%); $\mathrm{FA}=$ frequência absoluta (\%); $\mathrm{FR}=$ frequência relativa (\%); $\mathrm{CR}=$ cobertura relativa (\%); $\mathrm{VI}=$ valor de importância. Espécies ordenadas a partir do VI.

\begin{tabular}{|c|c|c|c|c|c|c|c|c|c|c|c|c|c|c|c|}
\hline \multirow{2}{*}{ Espécie } & \multirow{2}{*}{ Família } & \multirow[b]{2}{*}{$\begin{array}{l}\text { Voucher } \\
\text { MAR }\end{array}$} & \multirow[b]{2}{*}{$\begin{array}{l}\text { Formas } \\
\text { de Vida }\end{array}$} & \multicolumn{6}{|c|}{ Domínios Fitogeográficos } & \multirow{2}{*}{ NP } & \multirow{2}{*}{ CA } & \multirow{2}{*}{ FA } & \multirow{2}{*}{ FR } & \multirow{2}{*}{$\mathrm{CR}$} & \multirow{2}{*}{ VI } \\
\hline & & & & AM & CAA & $\mathrm{CR}$ & FA & PN & $\mathrm{PA}$ & & & & & & \\
\hline Paspalum maritimum Trin. & Poaceae & 7651 & $\mathrm{Hc}$ & $x$ & $x$ & $X$ & $x$ & & & 22 & $1586 ; 6$ & 44 & $7 ; 5$ & $46 ; 49$ & $54 ; 00$ \\
\hline Paspalum ligulare Nees & Poaceae & 7652 & $\mathrm{Hc}$ & $x$ & $x$ & & $x$ & & & 19 & 489.0 & 38 & 6.48 & 14.33 & 20.81 \\
\hline Cassytha filiformis L. & Lauraceae & 7653 & $\mathrm{Hp}$ & $x$ & $x$ & $X$ & $x$ & & & 28 & 372.8 & 56 & 9.55 & 10.92 & 20.47 \\
\hline $\begin{array}{l}\text { Chamaecrista hispidula (Vahl) } \\
\text { H.S. Irwin \& Barneby }\end{array}$ & Fabaceae & 7654 & $\mathrm{Te}$ & $x$ & $x$ & $x$ & $x$ & & & 36 & 230.5 & 72 & 12.28 & 6.75 & 19.03 \\
\hline $\begin{array}{l}\text { Euploca polyphylla (Lehm.) } \\
\text { J.I.M.Melo \& Semir }\end{array}$ & Boraginaceae & 7655 & $\mathrm{Hc}$ & $x$ & $x$ & & $x$ & & & 16 & 143.7 & 32 & 5.46 & 4.21 & 9.67 \\
\hline $\begin{array}{l}\text { Centrosema brasilianum (L.) } \\
\text { Benth. }\end{array}$ & Fabaceae & 7656 & $\mathrm{Tr}$ & $x$ & $x$ & $x$ & $x$ & $X$ & & 20 & 34.7 & 40 & 6.82 & 1.01 & 7.83 \\
\hline Borreria verticillata (L.) G. Mey. & Rubiaceae & 7657 & $\mathrm{Cf}$ & $x$ & $x$ & $X$ & $X$ & & & 15 & 28.8 & 30 & 5.12 & 0.84 & 5.96 \\
\hline $\begin{array}{l}\text { Chamaecrista flexuosa (L.) } \\
\text { Greene }\end{array}$ & Fabaceae & 7658 & $\mathrm{Te}$ & $x$ & $x$ & $x$ & $x$ & $X$ & & 12 & 51.8 & 24 & 4.09 & 1.51 & 5.6 \\
\hline Turnera pumilea L. & Turneraceae & 7659 & $\mathrm{Cf}$ & $x$ & $x$ & $x$ & $x$ & & & 13 & 12.6 & 26 & 4.43 & 0.37 & 4.8 \\
\hline Galactia striata (Jacq.) Urb. & Fabaceae & 7660 & $\mathrm{Tr}$ & $x$ & $x$ & $x$ & $x$ & & & 10 & 34.2 & 20 & 3.41 & 1.0 & 4.41 \\
\hline Pavonia cancellata (L.) Cav. & Malvaceae & 7661 & $\mathrm{Cf}$ & $x$ & $x$ & $x$ & $x$ & & & 11 & 21.1 & 22 & 3.75 & 0.62 & 4.37 \\
\hline Indeterminada & - & 7662 & - & $x$ & $x$ & $x$ & $x$ & & & 8 & 51.6 & 16 & 2.73 & 1.51 & 4.24 \\
\hline $\begin{array}{l}\text { Pombalia calceolaria (L.) } \\
\text { Paula-Souza }\end{array}$ & Violaceae & 7663 & $\mathrm{Cf}$ & $x$ & $\mathrm{x}$ & $X$ & $X$ & $X$ & & 9 & 4.3 & 18 & 3.07 & 0.12 & 3.19 \\
\hline Wedelia villosa Gardner & Asteraceae & 7664 & $\mathrm{Te}$ & & $x$ & $x$ & & & & 7 & 24.5 & 14 & 2.39 & 0.72 & 3.11 \\
\hline $\begin{array}{l}\text { Lepidaploa arenaria (Mart. ex } \\
\text { DC.) H.Rob. }\end{array}$ & Asteraceae & 7665 & $\mathrm{Te}$ & $x$ & & & $X$ & & & 6 & 29.7 & 12 & 2.04 & 0.87 & 2.91 \\
\hline Alternanthera tenella Colla & Amaranthaceae & 7666 & $\mathrm{Te}$ & $x$ & $x$ & $x$ & $x$ & $X$ & $x$ & 7 & 9.5 & 14 & 2.39 & 0.28 & 2.67 \\
\hline Clitoria laurifolia Poir. & Fabaceae & 7667 & $\mathrm{Cf}$ & $x$ & $x$ & $x$ & $x$ & & & 6 & 12.4 & 12 & 2.04 & 0.36 & 2.4 \\
\hline $\begin{array}{l}\text { Stylosanthes angustifolia } \\
\text { Vogel }\end{array}$ & Fabaceae & 7668 & $\mathrm{Te}$ & $x$ & $x$ & $x$ & $x$ & & & 6 & 11.5 & 12 & 2.04 & 0.34 & 2.38 \\
\hline $\begin{array}{l}\text { Marsypianthes chamaedrys } \\
\text { (Vahl) Kuntze }\end{array}$ & Lamiaceae & 7669 & $\mathrm{Te}$ & $x$ & $x$ & $X$ & $X$ & $X$ & & 6 & 6.9 & 12 & 2.04 & 0.20 & 2.24 \\
\hline Turnera subulata Sm. & Turneraceae & 7670 & $\mathrm{Cf}$ & $x$ & $x$ & $x$ & $x$ & & & 5 & 9.65 & 10 & 1.7 & 0.28 & 1.98 \\
\hline
\end{tabular}


Tabela 1. Continuação

\begin{tabular}{|c|c|c|c|c|c|c|c|c|c|c|c|c|c|c|c|}
\hline \multirow[b]{2}{*}{ Espécie } & \multirow[b]{2}{*}{ Família } & \multirow[b]{2}{*}{$\begin{array}{l}\text { Voucher } \\
\text { MAR }\end{array}$} & \multirow[b]{2}{*}{$\begin{array}{l}\text { Formas } \\
\text { de Vida }\end{array}$} & \multicolumn{6}{|c|}{ Domínios Fitogeográficos } & \multirow[b]{2}{*}{ NP } & \multirow[b]{2}{*}{ CA } & \multirow[b]{2}{*}{ FA } & \multirow[b]{2}{*}{$\mathrm{FR}$} & \multirow[b]{2}{*}{ CR } & \multirow[b]{2}{*}{$\mathrm{VI}$} \\
\hline & & & & AM & CAA & $\mathrm{CR}$ & FA & PN & PA & & & & & & \\
\hline $\begin{array}{l}\text { Ipomoea imperati (Vahl) } \\
\text { Griseb. }\end{array}$ & Convolvulaceae & 7671 & Cf & $X$ & & & $\mathrm{X}$ & & & 5 & 6.85 & 10 & 1.7 & 0.2 & 1.9 \\
\hline Mitracarpus salzmannianus DC. & Rubiaceae & 7672 & Cf & $\mathrm{X}$ & $x$ & $x$ & & & & 5 & 5.27 & 10 & 1.7 & 0.15 & 1.85 \\
\hline $\begin{array}{l}\text { Mimosa pudica var. tetrandra } \\
\text { (Humb. \& Bonpl. ex Willd.) DC. }\end{array}$ & Fabaceae & 7673 & Cf & $\mathrm{X}$ & $x$ & $x$ & $x$ & & & 3 & 17.2 & 6 & 1.02 & 0.50 & 1.52 \\
\hline $\begin{array}{l}\text { Asemeia martiana (A.W. } \\
\text { Benn.) J.F.B. Pastore \& J.R. } \\
\text { Abbott }\end{array}$ & Polygalaceae & 7674 & Cf & & $x$ & & $\mathrm{X}$ & & & 4 & 2.78 & 8 & 1.36 & 0.08 & 1.44 \\
\hline $\begin{array}{l}\text { Desmodium barbatum (L.) } \\
\text { Benth. }\end{array}$ & Fabaceae & 7675 & Cf & $\mathrm{X}$ & $x$ & $X$ & $x$ & $X$ & $X$ & 3 & 0.9 & 6 & 1.02 & 0.03 & 1.05 \\
\hline Waltheria indica L. & Malvaceae & 7676 & Cf & $x$ & $x$ & $x$ & $x$ & $x$ & & 2 & 0.6 & 4 & 0.68 & 0.017 & 0.69 \\
\hline Emilia fosbergii Nicolson & Asteraceae & 7677 & Cf & $\mathrm{X}$ & $x$ & $x$ & $x$ & $x$ & $x$ & 1 & 9 & 2 & 0.34 & 0.26 & 0.6 \\
\hline Mimosa candollei R. Grether & Fabaceae & 7678 & Cf & $x$ & $x$ & $x$ & $x$ & & & 1 & 2 & 2 & 0.34 & 0.06 & 0.4 \\
\hline Passiflora foetida L. & Passifloraceae & 7679 & $\operatorname{Tr}$ & $x$ & $x$ & $x$ & $x$ & $x$ & $x$ & 1 & 1 & 2 & 0.34 & 0.03 & 0.37 \\
\hline $\begin{array}{l}\text { Setaria vulpiseta (Lam.) } \\
\text { Roem. \& Schult. }\end{array}$ & Poaceae & 7680 & $\mathrm{Hc}$ & $\mathrm{X}$ & $x$ & $x$ & $x$ & $x$ & & 1 & 0.6 & 2 & 0.34 & 0.02 & 0.36 \\
\hline Zornia reticulata Sm. & Fabaceae & 7681 & Cf & $x$ & $\mathrm{x}$ & $x$ & $x$ & $x$ & $x$ & 1 & 0.5 & 2 & 0.34 & 0.01 & 0.35 \\
\hline Cnidoscolus urens (L.) Arthur & Euphorbiaceae & 7682 & Cf & $\mathrm{X}$ & $x$ & $X$ & $x$ & $x$ & & 1 & 0.25 & 2 & 0.34 & 0.007 & 0.34 \\
\hline $\begin{array}{l}\text { Borreria latifolia (Aubl.) K. } \\
\text { Schum. }\end{array}$ & Rubiaceae & 7683 & $\mathrm{Cf}$ & $X$ & $x$ & $x$ & $x$ & & $x$ & 1 & 0.03 & 2 & 0.34 & 0.0009 & 0.34 \\
\hline Cyperus sp 1 & Cyperaceae & 7684 & $\mathrm{Hc}$ & & & & & & & 1 & 0.02 & 2 & 0.34 & 0.0006 & 0.34 \\
\hline Cyperus sp 2 & Cyperaceae & 7685 & $\mathrm{Hc}$ & & & & & & & 1 & 0.02 & 2 & 0.34 & 0.0006 & 0.34 \\
\hline Total & & & & & & & & & & 50 & 3412.3 & 586 & 100 & 100 & 200 \\
\hline
\end{tabular}

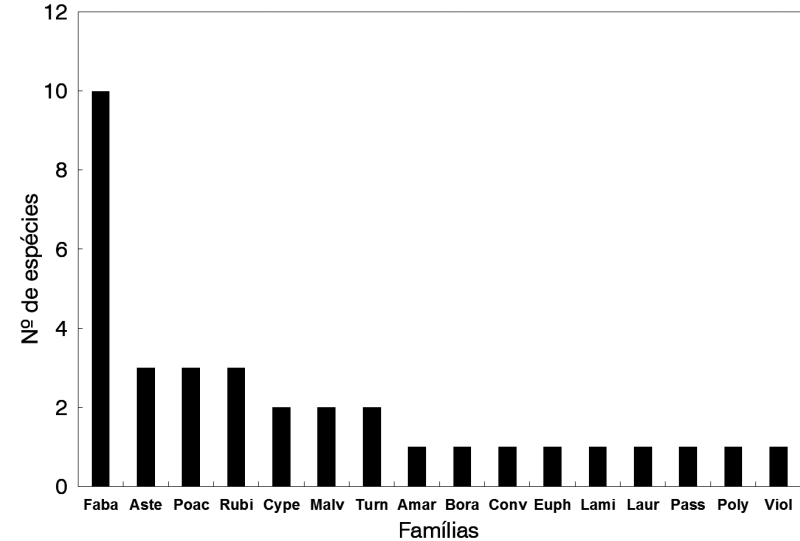

Figura 2. Distribuição do número de espécies por família, amostradas nas dunas da Praia de São Marcos, São Luís, Maranhão.

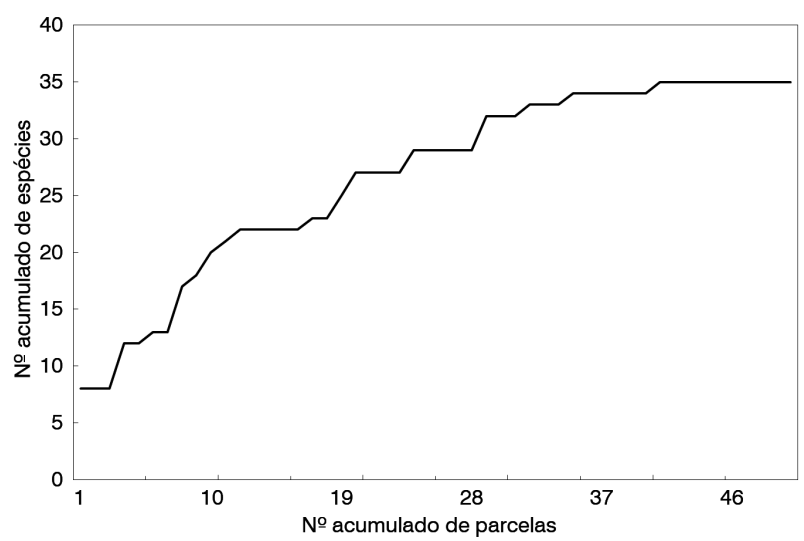

Figura 3. Curva de acumulação de espécies observadas em 50 parcelas (50 $\mathrm{m}^{2}$ ) amostradas nas dunas da Praia de São Marcos, São Luís, Maranhão. 
Chamaecrista hispidula (19\%) e Euploca polyphylla (9;6\%) (Tabela 1). As espécies Paspalum maritimum e Paspalum ligulare estiveram presentes em três dos cinco fragmentos amostrados e obtiveram os maiores VI's devido aos elevados valores de cobertura estimados.

A espécie Chamaecrista hispidula apresentou a maior frequência entre as espécies amostradas e Cassytha filiformis foi a segunda espécie mais frequente, porém obteve o terceiro maior valor de cobertura, alcançando o terceiro maior VI. Euploca polyphylla obteve valores inferiores aos das espécies citadas anteriormente para os parâmetros de frequência e cobertura, no entanto, tanto esta espécie quanto Chamaecrista hispidula estiveram presentes ao longo de toda área amostrada.

Quanto à estrutura da comunidade, quatro espécies foram consideradas dominantes, representando $57,1 \%$ do $\mathrm{VI}$, as intermediárias foram representadas por 21 espécies $(37,7 \%$ do VI) e as raras, por 10 espécies (2\% do VI), caracterizando uma estrutura oligárquica para a comunidade estudada.

Os índices de diversidade de Shannon ( $\left.\mathrm{H}^{\prime}\right)$ e equabilidade de Pielou (J'), a partir dos cálculos, apresentam dois valores: quando a cobertura foi utilizada como medida de abundância, obteve-se $\mathrm{H}_{\mathrm{c}}^{\prime}=1 ; 69$ nats $\mathrm{m}^{-2}$ e J' $=0 ; 47$. Quando a frequência foi utilizada como medida de abundância, obteve-se $\mathrm{H}_{\mathrm{f}}^{\prime}=3 ; 04$ nats $\mathrm{m}^{-2} \mathrm{eJ}_{\mathrm{f}}^{\prime}=0 ; 85$.

Quanto ao espectro biológico do estrato herbáceo foram encontradas cinco formas de vida: caméfitos, terófitos, hemicriptófitos, trepadeiras e holoparasitas (Tabela 1). Dentre estas, os caméfitos destacaram-se com 17 espécies perfazendo $50 \%$ do total amostrado, seguido por terófitos (sete espécies) e hemicriptófitos (seis espécies), representando juntos $38,2 \%$. Holoparasita foi representada somente por uma espécie (Figura 4).

Dos 35 táxons amostrados, apenas 32 foram considerados para a análise de distribuição fitogeográfica, visto que três

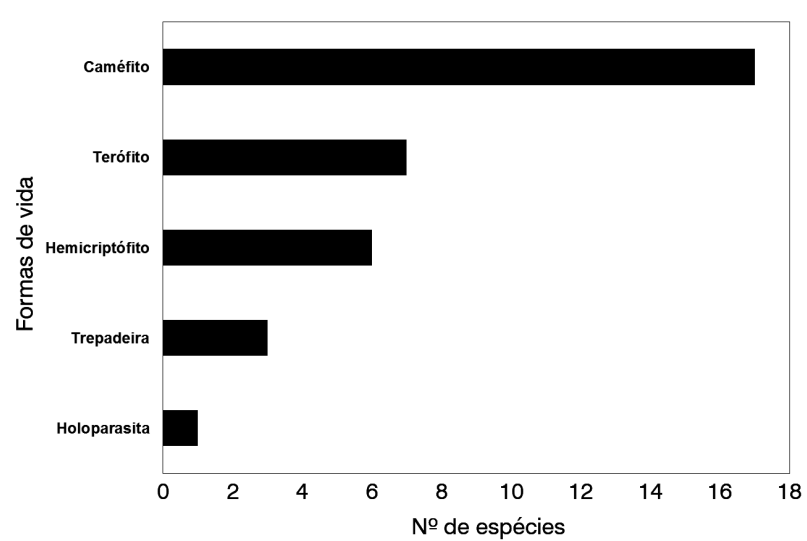

Figura 4. Distribuição das formas de vida em relação às espécies amostradas nas dunas da Praia de São Marcos, São Luís, Maranhão. táxons não foram identificados até espécie. Destes 32 táxons, a maioria apresentou ampla distribuição, com 15,6\% (cinco spp.) das espécies registradas em todos os biomas brasileiros; $25 \%$ (oito spp.) ocorre em cinco biomas e, aproximadamente, 37,5\% (12 spp.) dos táxons apresentou distribuição nos biomas Amazônia, Caatinga, Cerrado e Mata Atlântica. Apenas 21,9\% (sete spp.) do total amostrado apresenta ocorrência em menos de três biomas (Tabela 1).

Quanto ao status de conservação foram identificadas nas dunas da Praia de Sáo Marcos 14 categorias de impactos antrópicos, sendo encontrados com maior frequência: remoção da vegetação para construção de estradas costeiras; remoção da vegetação para construção de calçadas; ocupaçấo decorrente de urbanizaçáo; estabelecimento de residências e remoção da vegetação para instalação de quiosques comerciais.

Outros impactos ambientais que foram identificados, mas com menor frequência, foram: tráfego de carros sobre a vegetação de dunas; evidência da extração de espécies vegetais de interesse paisagístico; remoção da vegetação para estabelecer cultivos agrícolas (ex: macaxeira - Manihot esculenta Crantz e maxixe - Cucumis anguria L.); abertura de trilhas de pedestres para acesso à praia e criaçáo de estacionamento sobre a vegetação.

\section{DISCUSSÃO}

As dunas da Praia de São Marcos exibem longos trechos de área vegetada na costa de São Luís, cujo estrato herbáceo constitui uma vegetaçáo campestre de dunas, composta, em sua maioria, de ervas e subarbustos que atingem em média $40 \mathrm{~cm}$ de altura e apresentam longas faixas de gramínea nas dunas primárias. Estudos fitossociológicos realizados em outras áreas de dunas na costa brasileira (Cordeiro 2005; Palma e Jarenkow 2008; Menezes et al. 2012) apresentaram quantidade de espécies similares ao encontrado nas dunas do presente estudo. Cordeiro (2005) alocou 834 parcelas de $1 \mathrm{~m}^{2}$ divididas em três diferentes áreas nas dunas da Praia do Peró (RJ) e amostrou um total de 38 espécies herbáceas; Palma e Jarenkow (2008) em 34 parcelas de $4 \mathrm{~m}^{2}$ levantaram 15 espécies nas dunas frontais do Parque Estadual de Itapeva, no Rio Grande do Sul; Menezes et al. (2012) amostraram 28 espécies em 400 parcelas de $1 \mathrm{~m}^{2}$ nas dunas dos litorais norte e sul da Bahia. Considerando a diferença entre os tamanhos amostrais, observa-se um elevado número de espécies do estrato herbáceo das dunas do presente estudo, visto que foram registradas 35 espécies em 50 parcelas de $1 \mathrm{~m}^{2}$.

Em outras formaçóes vegetais da restinga (Pereira et al. 2004; Carvalho e Sá 2011) nota-se que o número de espécies encontradas para o estrato herbáceo também é semelhante, variando entre 33 e 39 espécies. 
As famílias que apresentaram maior riqueza específica (Fabaceae, Asteraceae, Poaceae e Rubiaceae) também se destacam em estudos de dunas (Cordeiro 2005; Palma e Jarenkow 2008; Menezes et al. 2012), além de trabalho florísticos nas restingas da regiáo Nordeste (Matias e Nunes 2001; Castro et al. 2012; Fernandes e Queiroz 2015) reiterando nossos resultados. Cabe ressaltar ainda que estas famílias comumente estáo entre as mais representativas em levantamentos fitossociológicos no estrato herbáceo de diversas fisionomias da restinga (Pereira et al. 2004; Klein et al. 2007; Carvalho e Sá 2011).

$\mathrm{Na}$ análise estrutural da vegetação, a estrutura oligárquica que caracteriza a área estudada é frequentemente observada no estrato herbáceo de dunas (Pereira et al. 2004; Cordeiro 2005; Palma e Jarenkow 2008). A oligarquia é comum em ambientes propensos a condiçóes ambientais extremas (Scarano 2002; 2009), uma vez que algumas espécies tolerantes a estresse ambiental, independentes de serem ou náo pioneiras, possibilitam o funcionamento da vegetação por propiciarem condiçôes para o estabelecimento de outras espécies mais exigentes.

No caso das dunas de Sáo Marcos, Paspalum maritimum e Paspalum ligulare apresentaram, nesta ordem, os maiores VI's (Tabela 1) ocasionados pela elevada cobertura. Ambas foram mais representativas na porção frontal das dunas cobrindo grandes extensóes e raramente aparecendo próximas, quando sob esta situação, Paspalum maritimum apresentava-se dominante.

Paspalum maritimum é descrita como uma espécie formadora de densas populaçóes (Oliveira et al. 2013) sendo um importante fixador de dunas (Leite e Andrade 2004) como foi constatado no presente estudo, sendo a espécie que obteve o maior valor de cobertura. Esta espécie também registrou maior VI nas dunas do Rio de Janeiro (Cordeiro 2005) conferindo este resultado à elevada capacidade desta planta fixar-se quando o solo já está ocupado e a movimentaçâo de areia é inconspícua. Paspalum ligulare, ao contrário de Paspalum maritimum, foi encontrada em áreas onde o solo ainda não estava completamente estabilizado e ocorreu espaçadamente ou em pequenas moitas isoladas em trechos com presença de pedregulho, ambiente similar ao descrito na literatura (Oliveira et al. 2013).

A presença dos rizomas em espécies da família Poaceae pode conferir certa vantagem na ocupaçáo da área de estudo por serem capazes de reter nutrientes e água (GütschowBento et al. 2010; Oliveira et al. 2013) possibilitando sua sobrevivência em dunas, além de conferirem maior resistência ao pisoteio, fogo, desmatamento e seca (Cirne et al. 2003; Maciel et al. 2009), impactos recorrentes no local.

Alguns autores alertam para o fato de espécies persistentes a perturbaçóes nas restingas poderem implicar na redução da diversidade local, já que, uma vez adaptadas a estas situaçóes, dominam extensas áreas limitando o estabelecimento de outras plantas e retardando o processo de sucessão (Santos et al. 2000; Cirne et al. 2003). Além disso, estudos evidenciam que Paspalum maritimum possui mecanismos de interferência na germinação e desenvolvimento de plantas adjacentes (Souza Filho 2006; Souza Filho e Mourão Jr. 2010). Estas características provavelmente estáo relacionadas ao baixo valor de cobertura de outras espécies que dividiam espaço com Paspalum maritimum e Paspalum ligulare.

Chamaecrista hispidula e Cassytha filiformis apresentaram os maiores valores de frequência e estão entre as cinco espécies com maiores VI para a área (ver Tabela 1). Ambas, junto à Euploca polyphylla foram notadas ao longo de toda área aparecendo desde a margem frontal da duna, onde são mais comuns, até as zonas mais interiores. Araújo et al. (2006) apontam as leguminosas, representada na área por Chamaecrista hispidula, como importantes fixadoras de nitrogênio, promovendo condiçôes edáficas favoráveis ao estabelecimento de espécies mais exigentes e consequentemente promovendo a regeneração natural da vegetação.

Euploca polyphylla é uma erva de hábito prostrado ou decumbente, em geral, bastante ramificada, com longos ramos difusos dispostos sobre o solo (Melo e Sales 2004; Melo e Semir 2010). Esta característica pode promover a redução da movimentação de areia e auxiliar na contenção da duna, salientando ainda que o hábito decumbente dessa espécie pode proporcionar sombreamento e favorecer um ambiente adequado para germinação de plantas mais exigentes.

O potencial estabilizador pioneiro foi verificado para Cassytha filiformis, uma vez que a espécie foi observada formando extensos "tapetes", característica também mencionada por Gomes e Guedes (2014) nas restingas da Bahia. No entanto, em áreas de cerrado, sua ocorrência é associada à indicação de perturbação antrópica (Santos e Alves 2012).

As análises de diversidade e equabilidade ainda são pouco utilizadas em estudos com o estrato herbáceo de dunas e restingas de um modo geral, visto a dificuldade de individualização de espécies com crescimento clonal, comum nesses ambientes (Pereira et al. 2004), impossibilitando a obtenção de valores de abundância. No entanto, seguindo a proposição de Magurran (1988), os estudos vêm utilizando valores de cobertura e frequência em substituição a esta medida, mas ainda não há um consenso quanto à eficiência destes parâmetros nestas análises e nem critérios que estabeleçam a escolha do melhor parâmetro a ser utilizado na representação da área, ficando a cargo do pesquisador.

Pereira et al. (2004) utilizando parâmetros de cobertura e frequência verificaram que valores de cobertura foram mais adequados por representarem melhor a estrutura do estrato herbáceo da formação aberta de Clusia, na restinga de Jurubatiba (RJ), tendo obtido $\mathrm{H}_{\mathrm{c}}{ }_{\mathrm{c}}=1,89$ nats $\mathrm{m}^{-2}$ e J' 
$=0,52$ (usando cobertura) e $\mathrm{H}_{\mathrm{f}}^{\prime}=3,01$ nats $\mathrm{m}^{-2}$ e J ${ }_{\mathrm{f}}=0,82$ (utilizando frequência). Carvalho e Sá (2011) encontraram diferenças mais sutis na comparaçáo destes parâmetros para a restinga de Massambaba (RJ) associando essa proximidade entre os valores à menor disparidade na proporção de cobertura entre as espécies, obtendo $\mathrm{H}^{\prime}=2,32$ nats $\mathrm{m}^{-2} \mathrm{e} \mathrm{J}$ ' $=0,67$ (usando cobertura) e $\mathrm{H}_{\mathrm{f}}^{\prime}=2,90$ nats $\mathrm{m}^{-2}$ e J ${ }_{\mathrm{f}}^{\prime}=0,84$ (utilizando frequência).

Para o estrato herbáceo na fisionomia de duna, Palma e Jarenkow (2008) no litoral norte do Rio Grande do Sul, utilizaram somente valores de frequência na obtenção da diversidade e equabilidade, e obtiveram $\mathrm{H}_{\mathrm{f}}^{\prime}=2,071$ nats $\mathrm{m}^{-2}$ e J' $=0,765$. No presente estudo, quando utilizada a cobertura foram obtidos baixos indicadores de diversidade $\left(\mathrm{H}_{c}^{\prime}=1,69\right.$ nats $\left.\mathrm{m}^{-2}\right)$ e equabilidade $\left(\mathrm{J}^{\prime}=0,47\right)$ e o inverso foi percebido na utilização de frequência em que altos valores foram obtidos para estes índices $\left(\mathrm{H}_{\mathrm{f}}^{\prime}=3,04\right.$ nats $\left.\mathrm{m}^{-2}, \mathrm{~J}_{\mathrm{f}}^{\prime}=0,85\right)$. As dunas do presente estudo apresentaram maior diversidade, ressaltandose que, a fim de comparaçáo, foram utilizados valores de frequência. Porém, levando-se em consideração a estrutura oligárquica da comunidade vegetal destas dunas, onde apenas quatro espécies respondem à mais da metade do VI $(57,1 \%)$, acredita-se que a utilizaçáo de valores do parâmetro de cobertura tenham correspondido de forma mais adequada à representação da diversidade da área de estudo, sustentando premissa de Pereira et al. (2004).

Em relaçáo às formas de vida analisadas para o estrato herbáceo, registrou-se o predomínio de caméfitos e terófitos (Tabela 1), semelhante ao encontrado em outras fisionomias campestres de vegetação de restinga (Almeida Jr. e Zickel 2009; Almeida Jr et al. 2009; Gomes e Guedes 2014). Já nos estudos que contemplaram áreas de dunas destacaram-se hemicriptófitas, geófitas e caméfitas (Pfadenhauer 1978; Palma e Jarenkow 2008). Embora neste estudo os hemicriptófitos tenham ocupado a terceira posição no ranking de formas de vida, estes apresentaram maior expressividade na área, especialmente por estarem representadas pelas espécies com os maiores valores de cobertura e consequentemente, maiores valores de importância (Paspalum maritimum e Paspalum ligulare). Cabe destacar que, mesmo considerando todas as formas de vida da área, entre elas os fanerófitos (os quais correspondem a 35 espécies, representando 30,4\% da riqueza de espécies do local - A.N.F. Silva, dados não publicados), a maior representatividade deve-se a composição herbácea, representada por caméfitos, terófitos e hemicriptófitos $(69,6 \%)$.

Caméfitos e terófitos possuem características que funcionam como mecanismos de escape a condiçôes climáticas desfavoráveis, sendo predominantes em habitats com características adversas (Caiafa e Silva 2005; Costa et al. 2007; Martins e Batalha 2011) assim como as dunas que apresentam vegetação com estratégias adaptativas que minimizam os fatores estressantes, possibilitando sua permanência (Leite e Andrade 2004). Todavia, a ausência de estudos que caracterizem a comunidade vegetal da restinga, a partir do espectro biológico, limita a realização de inferências interpretativas.

Entre as espécies registradas, nenhuma é restrita a um único bioma, assim como não há espécies exclusivas de restinga. Este comportamento generalista é marcante entre as espécies vegetais que ocorrem ao longo do litoral brasileiro (Assis et al. 2004; Silva et al. 2008).

A ausência de endemismos na restinga é atribuída à formaçáo geologicamente recente dessas planícies, favorecendo a ocupação por espécies provenientes de ambientes adjacentes que embora estejam submetidas a diferentes pressóes seletivas ainda não dispuseram de tempo suficiente para especiação (Rizzini 1979; Scarano 2002). Isto poderia explicar o grande número de espécies amostradas no presente estudo apresentar registro de ocorrência também nos biomas Amazônico, Caatinga, Cerrado e Mata Atlântica (78,1\% das espécies amostradas, tabela 1), somados a $15,6 \%$ de espécies que ocorrem em todos os biomas brasileiros (por exemplo, Alternanthera tenella, Desmodium barbatum, Emilia fosbegii, Passiflora foetida, Zornia reticulata).

Deve-se considerar ainda o posicionamento geográfico da área de estudo, que está situada numa faixa de transição entre a regiáo costeira da Amazônia oriental e a regiáo costeira do Semiárido nordestino. Essa regiāo costeira desenvolve-se próximo a diferentes domínios fitogeográficos, incluindo a Floresta Amazônica, o Cerrado e a Caatinga (Castro et al. 2012; Santos-Filho et al. 2013). Esta proximidade a biomas adjacentes pode influenciar a composiçáo florística (Scherer et al. 2005; Castro et al. 2012; Fernandes e Queiroz 2015), propiciando a formação de um complexo florístico-vegetacional característico que, submetido a um mosaico de condiçóes ambientais, permite a coexistência de espécies oriundas destes biomas, funcionando como um corredor ecológico (Castro et al. 2012). Desta forma, acredita-se que a localizaçáo das dunas pode ser um fator preponderante que influencie na composição florística (Fernandes e Queiroz 2015).

Em termos de conservaçáo, todos os fatores de impactos antrópicos presentes na lista de Rocha et al. (2003) foram observados na área de estudo, revelando forte antropizaçáo e condicionando urgência em medidas de conservação da área.

As dunas da Praia de Sáo Marcos constituem um ambiente bastante modificado, onde a vegetação de restinga deu lugar a grandes construçóes. Junto a isso, a implantação e ampliação da avenida ao longo da orla marítima também ocasionou grande perda da vegetaçáo de dunas (observaçáo 
dos autores). Além disso, ao longo de toda a extensão da área é possível observar, em muitos pontos, grandes estabelecimentos comerciais (restaurantes, hotéis, lojas) e edifícios residenciais que já comprometeram grande parte da flora estabelecida sobre estas dunas, causando a redução e descaracterização da vegetação (observação dos autores).

$\mathrm{Na}$ área de estudo também se nota a presença constante de queimadas intencionais e/ou acidentais, impacto que embora esteja ausente nas categorias propostas por Rocha et al. (2003), afeta intensamente a vegetaçáo de restinga ao longo do ano, especialmente no período seco que compreende os meses de julho a dezembro (observaçáo dos autores). Ressalta-se aqui a ausência de estudos que façam delineamentos da situaçáo ambiental de áreas de dunas na costa brasileira, gerando a impossibilidade de comparaçóes entre áreas.

É importante destacar que, dependendo da intensidade da perturbação, a vegetaçáo de restinga apresenta potencial para regeneraçáo natural, porém ainda não se sabe como áreas intensamente impactadas respondem ao processo de recuperação (Marques et al. 2015) ressaltando-se que o solo das restingas, pobre em nutrientes e com elevado grau de salinidade, dificulta a recomposição da vegetação (Rocha et al. 2003).

\section{CONCLUSÕES}

Nossos resultados mostraram uma riqueza de espécies herbáceas expressiva quando comparada a outras áreas de dunas do litoral brasileiro, embora esta vegetação de restinga já tenha sido fortemente reduzida no local. Além disso, foram amostradas espécies com características essenciais para a fixação das dunas, podendo favorecer a manutenção da área ou dificultar o estabelecimento e/ou desenvolvimento de outras espécies. No entanto, diversas fontes de impactos ao longo da orla promovem a reduçáo, fragmentação e descaracterização da vegetação estabelecida, dificultando o equilíbrio do ecossistema. Diante disso, tornam-se imprescindíveis novas abordagens que somem esforços para viabilizar a implantaçâo de medidas ambientais urgentes que promovam à proteção dos remanescentes de vegetação litorânea e/ou tracem estratégias de uso sustentável pela população, assegurando a proteçấo da biodiversidade da área que ainda está extremamente vulnerável à depredação.

\section{AGRADECIMENTOS}

Ao Conselho Nacional de Desenvolvimento Científico e Tecnológico (CNPq), pela bolsa do primeiro autor, à Fundação de Amparo à Pesquisa e Desenvolvimento Científico do Maranhão (FAPEMA), pelo financiamento do projeto. Ao Laboratório de Estudos Botânicos da Universidade Federal do Maranhão pelo apoio e infraestrutura para realizaçáo desta pesquisa, e a David Barros Muniz pela elaboração do mapa.

\section{BIBLIOGRAFIA CITADA}

Almeida Jr., E.B.; Olivo, M.A.; Araújo, E.L.; Zickel, C.S. 2009. Caracterização da vegetação de restinga da RPPN de Maracaípe, Pernambuco, com base na fisionomia, flora, nutrientes do solo e lençol freático. Acta Botanica Brasilica, 23: 36-48.

Almeida Jr., E.B.; Zickel, C.S. 2009. Fisionomia psamófila-reptante: riqueza e composição de espécies na praia da pipa, rio grande do norte, Brasil. Pesquisas, Botanica, 60: 289-299.

Almeida, J.R.; Suguio, K. 2012. Potencialidade geoturística das dunas eólicas da Ilha Comprida-Estado de Sáo Paulo. Geociências, 31: 473-484

APG III. 2009. An update of the Angiosperm Phylogeny Group classification for the orders and families of flowering plants: APG III. Botanical Journal of the Linnean Society, 141: 399-436.

Araújo, F.S.; Martins, S.V.; Meira Neto, J.A.A.; Lani, J.L.; Pires, I.E. 2006. Estrutura da vegetação arbustivo-arbórea colonizadora de uma área degradada por mineração de caulim, Brás Pires, MG. Revista Árvore, 30: 107-116.

Assis, A.M.; Thomaz, L.D.; Pereira, O.J. 2004. Florística de um trecho de floresta de restinga no município de Guarapari, Espírito Santo, Brasil. Acta Botanica Brasilica, 18: 191-201.

Brower, J.E.; Zar, J.H. 1977. Field and laboratory methods for general ecology. Wm. C. Brown Co, Iowa, 194p.

Cabral-Freire, M.C.C.; Monteiro, R. 1993. Florística das praias da Ilha de São Luis, Estado do Maranhão (Brasil): Diversidade de espécies e suas ocorrências no litoral brasileiro, Acta Amazonica, 23: $125-140$.

Cabral-Freire, M.C.C.; Monteiro, R. 1994. Praias e dunas da Ilha de São Luis, estado do Maranhão (Brasil): florística e topografia. Arquivos de Biologia e Tecnologia, 37: 865-876.

Caiafa, A.N.; Silva, A.F. 2005. Composição florística e espectro biológico de um campo de altitude no Parque Estadual da Serra do Brigadeiro, Minas Gerais - Brasil. Rodriguesia, 56: 163-173.

Carboni, M.; Carranza, M.L.; Acosta, A. 2009. Assessing conservation status on coastal dunes: A multiscale approach. Landscape and Urban Planning, 91: 17-25.

Carvalho, D.A.; Sá, C.F.C. 2011. Estrutura do estrato herbáceo de uma restinga arbustiva aberta na APA de Massambaba, Rio de Janeiro, Brasil. Rodriguésia, 62: 367-378.

Castro, A.S.F.; Moro, M.F.; Menezes, M.O.T. 2012. O Complexo Vegetacional da Zona Litorânea no Ceará: Pecém, São Gonçalo do Amarante. Acta Botanica Brasilica, 26: 108-124.

Chaves, A.D.C.G.; Santos, R.M.S.; Santos, J.O.; Fernandes, A.A.; Maracajá, P.B. 2013. A importância dos levantamentos florístico e fitossociológico para a conservação e preservação das florestas. Revista Agropecuaria Cientifica no Semi-Árido, 9: 42-48.

Cirne, P.; Zaluar, H.L.T.; Scarano, F.R. 2003. Plant diversity, interspecific associations, and postfire resprouting on a sandy spit in a brazilian coastal plain. Ecotropica, 9: 33-38.

Citadini-Zanette, V.; Baptista, L.R.M. 1989. Vegetação herbácea terrícola de uma comunidade florestal em Limoeiro, Município de Torres, Rio Grande do Sul. Boletim do Instituto de Biociências, 45: $1-87$. 
Cordazzo, C.V.; Paiva, J.B.; Seeliger, U. 2006. Plantas de dunas da costa sudeste atlântica. Useb, Pelotas, $107 \mathrm{p}$.

Cordeiro, S.Z. 2005. Composição e distribuição florística da vegetação herbácea em três áreas com fisionomias distintas na Praia do Peró, Cabo Frio, RJ, Brasil. Acta Botanica Brasilica, 19: 679-693.

Costa, R.C.; Araújo, F.S.; Lima-Verde, L.W. 2007. Flora and lifeform spectrum in an area of deciduous thorn woodland (caatinga) in northeastern, Brazil. Journal of Arid Environments, 68: 11-22.

Danilevicz, E.; Janke, H.; Pankowski, L.H.S. 1990. Florística e estrutura da comunidade herbácea e arbustiva da Praia do Ferrugem, Garopaba, SC. Acta Botanica Brasilica, 4:21-34.

Falkenberg, D.B. 1999. Aspectos da flora e da vegetação secundária da restinga de Santa Catarina, sul do Brasil. Insula, 28: 1-30.

Fernandes, M.F.; Queiroz, L.P. 2015. Floristic surveys of Restinga Forests in southern Bahia, Brazil, reveal the effects of geography on community composition. Rodriguésia, 66: 51-73.

Gomes, F.S.; Guedes, M.L.S. 2014. Flora vascular e formas de vida das formaçóes de restinga do litoral norte da Bahia, Brasil. Acta Biológica Catarinense, 1: 22-43.

Gütschow-Bento, L.H.; Castellani, T.T.; Lopes, B.C.; Godinho, P.S. 2010. Estratégia de crescimento clonal e fenologia de Syngonanthus chrysanthus Ruhland (Eriocaulaceae) nas baixadas entre dunas da Praia da Joaquina, Florianópolis, SC, Brasil. Acta Botanica Brasilica, 24: 205-213.

Inacio, C.D.; Jarenkow, J.A. 2008. Relaçôes entre a estrutura da sinúsia herbácea terrícola e a cobertura do dossel em floresta estacional no sul do Brasil. Revista Brasileira de Botanica, 31: 41-51.

Klein, A.S.; Citadini-Zanette, V.; Santos, R. 2007. Florística e Estrutura comunitária de restinga herbácea no município de Araranguá, Santa Catarina. Biotemas, 20: 15-26.

Köppen, W. 1948. Climatologia: con un estudio de los climas de la tierra.1a ed. Fondo de Cultura Economia, México, 478p.

INMET, 2015. Climatologia (http://www.inmet.gov.br/portal/index. php?r=clima/mesTempo). Acesso em: 29/05/2015.

Leite, A.V.L.; Andrade, L.H.C. 2004. Riqueza de espécies e composição florística em um ambiente de duna após 50 anos de pressão antrópica: um estudo na Praia de Boa Viagem, Recife, PE - Brasil. Biotemas, 17: 29-46.

Lista de espécies da flora do Brasil, 2015. Jardim Botânico do Rio de Janeiro (www.floradobrasil.jbrj.gov.br). Acesso em: 20/04/2015.

Maciel, J.R.; Oliveira, R.C.; Alves, M. 2009. Paspalum L. (Poaceae: Panicoideae: Paniceae) no estado de Pernambuco, Brasil. Acta Botanica Brasilica, 23: 1145-1161.

Magurran, A.E. 1988. Ecological diversity and itsmeasurement. Princeton University Press, New Jersey, 179p.

Marques, M.C.M.; Silva, S.M.; Liebsch, D. 2015. Coastal plain forests in southern and southeastern Brazil: ecological drivers, floristic patterns and conservation status. Brazilian Journal of Botany, 38: 1-18.

Martins, F.R.; Batalha, M.A. 2011. Formas de vida, espectro biológico de Raunkiaer e fisionomia da vegetação. In: Felfili, J.M.; Eisenlohr, P.V.; Melo, M.M.R.F.; Andrade, L.A.; Meira
Neto, J.A.A. (Ed). Fitossociologia no Brasil: métodos e estudos de caso. v. 1. Editora da Universidade Federal de Viçosa, Viçosa, Minas Gerais, p. 44-85.

Matias, L.Q.; Nunes, E.P. 2001. Levantamento florístico da área de Proteção Ambiental de Jericoacoara, Ceará. Acta Botanica Brasilica, 15: 35-43.

Melo, J.I.M.; Sales, M.F. 2004. Heliotropium L. (Boraginaceae - Heliotropioideae) de Pernambuco, Nordeste do Brasil. Rodriguésia, 55: 65-87.

Melo, J.I.M.; Semir, J. 2010. Taxonomia do gênero Euploca Nutt. (Heliotropiaceae) no Brasil. Acta Botanica Brasilica, 24: 111-132.

Menezes, C.M.; Espinheira, M.J.C.L.; Dias, F.J.K.; Silva, V.I.S. 2012. Composição florística e fitossociologia de trechos da vegetação praial dos litorais norte e sul do Estado da Bahia. Revista Biociências, 18: 35-41.

Mori, L.A.; Silva, L.A.M.; Lisboa, G.; Coradin, L. 1989. Manual de manejo do herbário fanerogâmico. Centro de Pesquisa do Cacau, lhéus, $97 \mathrm{p}$.

Mueller-Dombois, D.; Ellenberg, H. 1974. Aims and methods of vegetation ecology. Wiley, New York, 547p.

Munhoz, C.B.R.; Araújo, G.M. 2011. Métodos de Amostragem do Estrato Herbáceo subarbustivo. In: Felfili, J.M.; Eisenlohr, P.V.; Melo, M.M.R.F.; Andrade, L.A.; Meira Neto, J.A.A. (Ed). Fitossociologia no Brasil: métodos e estudos de caso. v. 1. Editora da Universidade Federal de Viçosa, Viçosa, Minas Gerais, p. 213-230.

Oliveira, A.C.C.A.; Sousa, R.M. 2011. Ecodinâmica dos sistemas dunares do município de Pirambu, Litoral Norte de Sergipe. Sociedade e Território, 23: 2-20.

Oliveira, R.C.; Santana, S.H.; Silva, A.S.; Maciel, J.R.; Valls, J.F.M. 2013. Paspalum (Poaceae) no Rio Grande do Norte, Brasil. Rodriguésia, 64: 847-862.

Palma, C.B.; Jarenkow, J.A. 2008. Estrutura de uma formação herbácea de dunas frontais no litoral norte do Rio Grande do Sul, Brasil. Biociências, 16: 114-124.

Pereira, M.C.A.; Cordeiro, S.Z.; Araujo, D.S.D. 2004. Estrutura do estrato herbáceo na formação aberta de Clusia do Parque Nacional da Restinga de Jurubatiba, RJ, Brasil. Acta Botanica Brasilica, 18: 677-687.

Pfadenhauer, J. 1978. Contribuição ao conhecimento da vegetação e de suas condiçóes de crescimento nas dunas costeiras do Rio Grande do Sul, Brasil. Revista Brasileira de Biologia, 38: 827-836.

Raunkiaer, C. 1934. Life forms and terrestrial plant geography. Claredon Press, Oxford, 137p.

Rizzini, C.T. 1979. Tratado de Fitogeografia do Brasil. HUCITEC EDUSP, São Paulo, 374p.

Rocha, C.F.D.; Bergallo, H.G.; Alves, M.A.S.; Van Sluys, M. 2003. A Biodiversidade nos Grandes Remanescentes Florestais do Estado do Rio de Janeiro e nas Restingas da Mata Atlântica. Instituto Biomas \& Conservation International Brasil, Editora Rima, p. 160.

Santos, C.R.; Medeiros, J.D. 2003. A ocupaçáo humana das áreas de preservação permanente (vegetaçáo fixadora de dunas) das 
localidades das Areias do Campeche e Morro das Pedras, Ilha de Santa Catarina, SC. Revista de Estudos Ambientais, 5: 22-41.

Santos, M.; Rosado, S.C.S.; Oliveira-Filho, A.T.; Carvalho, D. 2000. Correlaçóes entre variáveis do solo e espécies herbáceoarbustivas de dunas em revegetação no litoral norte da Paraíba. Cerne, 6: 19-29.

Santos, S.O.; Alves, M. 2012. Flora da Usina São José, Igarassu, Pernambuco: Lauraceae. Rodriguésia, 63: 689-703.

Santos-Filho, F.S.; Almeida Jr., E.B.; Soares, C.J.R.S. 2013. Cocais: zona ecotonal natural ou artificial? Revista Equador, 1: 02-13.

Scarano, F.R. 2002. Structure, function and floristic relantioships of plants communities in stressful habitats marginal to Brazilian Atlantic Rainforest. Annals of Botany, 90: 517-524.

Scarano, F.R. 2009. Plant communities at the periphery of the Atlantic rain forest: Rare-species bias and its risks for conservation. Biological Conservation, 142: 1201-1208.

Scherer, A.; Maraschin-Silva, F.; Baptista, L.R.M. 2005. Florística e estrutura do componente arbóreo de matas de restinga arenosa no Parque Estadual de Itapuã, RS, Brasil. Acta Botanica Brasilica, 19: 717-727.
Silva, S.M. 1999. Diagnóstico das restingas do Brasil. Fundação Bio Rio (ed.), Workshop Avaliação e Açóes Prioritárias para a Conservação da Biodiversidade da Zona Costeira. Ilhéus, Bahia, 30 p.

Silva, Q.D. 2012. Mapeamento geomorfológico da Ilha do Maranhão. Tese de doutorado, Universidade Estadual Paulista, Presidente Prudente, São Paulo. 249p.

Silva, S.S.L.; Zickel, C.S.; Cestaro, L.A. 2008. Flora vascular e perfil fisionômico de uma restinga no litoral sul de Pernambuco, Brasil. Acta Botanica Brasilica, 22: 1123-1135.

Souza Filho, A.P.S. 2006. Interferência potencialmente alelopática do capim gengibre (Paspalum maritimum) em áreas de pastagens cultivadas. Planta Daninha, 24: 451-456.

Souza Filho, A.P.S.; Mourão Jr., M. 2010. Padrão de resposta de Mimosa pudica e Senna obtusifolia à atividade potencialmente alelopática de espécies de Poaceae. Planta Daninha, 28: 927-938.

Veloso, H.P.; Rangel Filho, A.L.R.; Lima, J.C.A. 1991. Classificação da vegetaçáo brasileira adaptada a um sistema universal. IBGE, Departamento de Recursos Naturais e Estudos Ambientais, Rio de Janeiro, $124 \mathrm{p}$.

Recebido em 23/09/2015

Aceito em 24/02/2016 
